\title{
A RELAÇÃO ENTRE MÉTODOS DE ENSINO E ESTILOS DE APRENDIZAGEM LEAN MANUFACTURING: UMA PESQUISA EMPÍRICA SOBRE UM CURSO DE PÓS-GRADUAÇÃO EM ENGENHARIA DE PRODUÇÃO
}

\section{RELATIONSHIP BETWEEN LEAN MANUFACTURING TEACHING METHODS AND LEARNING STYLES: AN EMPIRICAL RESEARCH ON A PRODUCTION ENGINEERING POSTGRADUATE COURSE}

\author{
Guilherme L. Tortorella* E-mail: g.tortorella@ufsc.br \\ Rogério F. Miorando*E-mail: Rogerio.miorando@ufsc.br \\ Diego C. Fettermann* E-mail: d.fettermann@ufsc.br \\ *Universidade Federal de Santa Catarina (UFSC), Florianópolis, SC, Brasil
}

\begin{abstract}
Resumo: Devido a uma crescente concorrência mundial em relação ao desempenho operacional e lucros empresariais, a Lean Manufacturing (LM) tem sido uma matéria emergente e relevante entre os cursos de pós-graduação em Engenharia de Produção e Gestão de Negócios. Apesar dos avanços no ensino de princípios e técnicas de LM, o caráter prático inerente ao LM mina a aprendizagem e o desenvolvimento dos alunos. Nesse sentido, este estudo visa propor uma metodologia para aprimorar o aprendizado de LM de graduados em Engenharia de Produção de um curso de pós-graduação stricto sensu. A metodologia proposta combina os métodos tradicionais de ensino com a abordagem PBL (abordagem baseada em problemas), baseada em problemas reais de empresas em fase de implementação lean. O método é ilustrado com um exemplo em uma universidade pública brasileira, que foi um dos precursores no ensino de Engenharia de Produção no Brasil em nível de pós-graduação. Os resultados indicam que o PBL pode ser um método complementar eficaz para o aprendizado de LM, especialmente se os estudantes de pós-graduação são expostos a problemas reais em empresas que estão passando por uma implementação lean e relacionam isto com o corpo atual da literatura.
\end{abstract}

Palavras-chave: Métodos tradicionais de ensino. Aprendizagem baseada em problemas. Índice de estilo de aprendizagem. Produção enxuta.

\begin{abstract}
The interest in improving the quality in engineering education is widely deemed. Due to an increasingly worldwide competition regarding operational performance and business profits, Lean Manufacturing (LM) has been an emerging and relevant subject among Production Engineering and Business Management postgraduate courses. Despite the advances in teaching LM principles and techniques, the practical character inherent to LM undermines learning and development of students. In this sense, this study aims at proposing a methodology to enhance LM learning of Production Engineering graduates of a stricto sensu postgraduate course. The proposed methodology combines traditional teaching methods to PBL (problem-based learning) approach based on real problems of companies undergoing a lean implementation. The method is illustrated with an example in a Brazilian public university, which was one of the precursors in teaching Production Engineering in Brazil at postgraduate level. The findings indicate that PBL may be an effective complementary method for LM learning, especially if graduate students are exposed to real problems in companies that are undergoing a lean implementation and related it to the current body of the literature.
\end{abstract}

Keywords: Traditional teaching methods. Problem-based learning. Index of learning style. Lean manufacturing. Postgraduate engineering education. 


\section{INTRODUÇÃO}

Os alunos podem aprender de várias maneiras, como ouvir, praticar, visualizar, etc. Entretanto, os métodos de ensino também estão sujeitos a variações. Por exemplo, alguns instrutores preferem discussões ou demonstrações, enquanto outros adotam palestras (KALISKÁ, 2012). Neste sentido, a eficácia da aprendizagem é governada em parte pela habilidade nativa dos alunos, sua preparação prévia e também pela compatibilidade de suas preferências de aprendizagem com os métodos de ensino aplicados (FELDER; SILVERMAN, 1988; FELDER; SPURLIN, 2005). Os desajustes entre as preferências de aprendizagem dos alunos e os métodos de ensino dos professores resultam em estudantes entediados e desatentos que tendem a se comportar mal em testes e, em última instância, mudar para outros currículos (LITZINGER et al., 2007). Por outro lado, abordagens extremas, como a instrução individualizada completa e a abordagem de tamanho único, são impraticáveis ou ineficazes para a maioria dos estudantes (RAMSDEN, 2003; CAMPOS et al., 2013).

Dentro do contexto do ensino de engenharia, o crescente desinteresse dos alunos nos últimos anos tem levado a uma redução íngreme de inscrição em muitos programas e cursos de pós-graduação (FELDER; BRENT, 2005; GALAND et al, 2012.). Além disso, um desafio comum no ensino da engenharia é determinar como apresentar o material do curso para que os estudantes não só adquiram conhecimentos, mas também tornar-se aprendizes autônomos que desenvolvam habilidades de resolução de problemas que podem ser aplicados em suas carreiras (WHITE, 2001; SHEPPARD et al., 2008). Portanto, oferecer métodos de ensino complementares que melhorem o ensino de engenharia e busquem acomodar as diversas necessidades dos alunos em uma classe pode fornecer melhores resultados de aprendizagem, motivando os alunos sobre os seus cursos (BIGGS, 2003; BEDDOES et al., 2010).

Especificamente para a educação em Engenharia de Produção, os princípios, conceitos e técnicas de Lean Manufacturing (LM) têm sido amplamente considerados como diferenciais para as empresas que alcançam um melhor desempenho operacional (LIKER, 2004; SHAH; WARD, 2007). LM é uma abordagem organizacional e estratégica que visa eliminar atividades sem valor agregado para 
oferecer um produto ou serviço com custo mínimo e melhor qualidade de acordo com a expectativa dos clientes (LIKER; MEIER, 2006; WOMACK; JONES, 2009).

A falta de experiência prática dos alunos de pós-graduação e a aplicação única de métodos de ensino tradicional obriga a uma modificação dos estilos de ensino e aprendizagem, a fim de compensar tais lacunas e desajustes. Com base nesses argumentos, duas questões de pesquisa podem ser levantadas e descritas como:

1)Qual é a associação entre os métodos de ensino e os estilos de aprendizagem para a educação LM?

2) Existe uma associação mais eficaz para o ensino de LM?

Assim, este artigo tem como objetivo identificar os métodos de ensino que melhor correspondem aos estilos de aprendizagem de um curso de pós-graduação em lean manufacturing. Para isso, foram coletados dados de 15 estudantes de um curso de pós-graduação stricto sensu em engenharia de produção que oferece duas disciplinas de LM com diferentes métodos de ensino: (i) abordagem de aprendizagem baseada em problemas (PBL) em problemas reais de empresas submetidas a uma implementação lean; e (ii) métodos de ensino tradicionais, tais como palestras, estudos de caso e discussões em sala de aula. As preferências de aprendizagem dos alunos foram colhidas através da aplicação do questionário Index of Learning Styles (ILS) (FELDER; SOLOMAN, 2004) e seus desempenhos de aprendizagem foram avaliados no final de cada disciplina. Os dados coletados foram analisados por meio de técnicas multivariadas. Os resultados mostram como as diferentes preferências de aprendizagem dos alunos correspondem a diferentes métodos de ensino para o aprendizado de LM, proporcionando maior desempenho dos alunos.

Este estudo pretende contribuir para o preenchimento de uma lacuna observada na literatura no que se refere ao ensino e aprendizagem da lean manufacturing, pois permite a identificação da associação mais efetiva para o ensino de LM em nível de pós-graduação. Além de sua contribuição teórica, esta pesquisa fornece implicações práticas que podem auxiliar professores e instrutores a aproximar-se melhor dos métodos de ensino de LM que são mais prováveis de ser eficazes de acordo com as preferências de aprendizagem dos alunos. O restante deste artigo está estruturado da seguinte forma. A Seção 2 apresenta uma visão geral da literatura sobre métodos de ensino e aprendizagem de LM. A seção 3 descreve o método proposto, com os resultados de sua aplicação apresentados na seção 4 . A 
seção 5 encerra o artigo apresentando conclusões e oportunidades de pesquisa futuras.

\section{REVISÃO DE LITERATURA}

\subsection{Ensino e aprendizagem de LM}

De acordo com Liker (2004), os quatro princípios principais de um sistema LM são: (i) filosofia de pensamento a longo prazo; (ii) melhoria contínua do processo para eliminar desperdício; (iii) respeito, desafio e crescimento das pessoas e parceiros; e (iv) resolução de problemas através da melhoria contínua e aprendizagem. Em geral, os princípios de LM referem-se direta ou indiretamente à necessidade de aprendizagem em todos os níveis da organização (TORTORELLA; FOGLIATTO, 2014), sendo desenvolvidos principalmente com base em um sistema de aprendizado por meio de um método estruturado e científico (SPEAR; BOWEN, 1999). Este sistema de aprendizagem foi seguido por muitas empresas de manufatura e serviços que estão sob a implementação lean, transformando os funcionários em pensadores e aprendizes verdadeiramente ativos (ALVES et al., 2012; TORTORELLA et al., 2015).

No contexto acadêmico, o ensino de princípios e técnicas de LM para os alunos tem sido considerado imperativo, devido ao cenário desafiador e competitivo que a maioria das organizações enfrenta atualmente e demanda de sua força de trabalho (MURMAN et al., 2007). Assim, a LM tem sido integrada de forma multidisciplinar em várias universidades como parte dos currículos educacionais, com especial ênfase na gestão empresarial e na educação em engenharia (ALVES et al., 2014). Um exemplo dessa integração é a criação da LEAN (Lean Education Academic Network), que é um grupo de educadores universitários que procuram promover a educação lean na academia dos EUA através da partilha de conhecimentos e materiais de ensino (FLIEDNER; MATHIESON, 2009). Alves et al. (2014) e Flumerfelt et al. (2014) introduzem o conceito de educação em engenharia enxuta (LEE), que é definida como uma abordagem sistemática, centrada no aluno e valorizada para a prestação de serviços educacionais que permite aos estudantes atender, liderar e moldar holisticamente as necessidades industriais, individuais e sociais integrando compreensão e aplicação de ferramentas e conceitos de engenharia. 
Outra tendência de ensino compreende o estabelecimento de parcerias entre universidades e indústrias para ensinar e aplicar ideias lean. Por exemplo, a Universidade de Kettering estabeleceu um programa de LM em conjunto com a Ford Motor Company, levando a um Mestrado em Ciências de Engenharia ou Engenharia de Manufatura (KETTERING UNIVERSITY, 2016). Além disso, a Universidade de Kentucky promove o Lean Systems Program chamado True Lean (UNIVERSIDADE DE KENTUCKY, 2016), que certifica os alunos através de atividades práticas cujos instrutores são ex-funcionários da Toyota. Martens et al. (2010) e Carvalho et al. (2013) descrevem a Lean Learning Academy, um projeto que abrange universidades e empresas holandesas, suecas, polonesas, portuguesas e romenas, a fim de proporcionar um programa de formação inovador sobre LM.

No entanto, os livros tradicionais de LM e os métodos de ensino dependem fortemente da experiência de manufatura. Conger e Miller (2013) afirmam que a aplicação isolada de um método de ensino, como estudos de caso por exemplo, pode ser uma má escolha para o ensino de LM, uma vez que podem faltar os alunos tanto a base física como conceitual do conhecimento. Além disso, existem outras habilidades fundamentais necessárias para a implementação real de LM que são difíceis de serem ensinadas a partir de um livro. Várias pesquisas relatam cursos e programas lean que utilizam diferentes metodologias de ensino, tais como palestras com convidados em sala de aula, jogos e simulações virtuais, análise de estudos de caso, visitas a plantas, entrevistas com especialistas, etc. (MURAN et al., 2012; CONGER; MILLER, 2014; KANIGOLLA et al., 2014).

A Tabela 1 consolida alguns dos principais métodos evidenciados na literatura para o ensino de LM. Além disso, a Tabela 1 descreve resumidamente os objetivos e achados dos autores com base na aplicação dos métodos propostos. 
Tabela 1 - Consolidação dos principais métodos de ensino para educação LM

\begin{tabular}{|c|c|c|c|}
\hline Referência & Objetivos & Método de ensino & Resultados \\
\hline 1 & $\begin{array}{l}\text { Desenvolver a capacidade dos alunos } \\
\text { para aplicar princípios de design lean; } \\
\text { desenvolver a capacidade dos alunos } \\
\text { para analisar dados; e aumentar a } \\
\text { compreensão do aluno da dinâmica e } \\
\text { variabilidade fundamentais do } \\
\text { processo. }\end{array}$ & $\begin{array}{l}\text { Exercícios de laboratório } \\
\text { lean, baseados em uma } \\
\text { simulação física da } \\
\text { montagem de um relógio. }\end{array}$ & $\begin{array}{l}\text { Os alunos que fizeram o curso com os exercícios } \\
\text { de laboratório expressaram significativamente } \\
\text { mais confiança em sua capacidade de } \\
\text { compreender e aplicar ideias lean, bem como } \\
\text { para calcular as medidas do processo. }\end{array}$ \\
\hline 2 & $\begin{array}{l}\text { Complementar a compreensão da } \\
\text { teoria lean para que os alunos se } \\
\text { tornem capazes de aplicá-la fora da } \\
\text { sala de aula. }\end{array}$ & $\begin{array}{l}\text { Integrar PBL na forma de } \\
\text { um projeto para um cliente } \\
\text { externo à abordagem de } \\
\text { montagem de veículos } \\
\text { Lego desenvolvida em } \\
\text { sala de aula. }\end{array}$ & $\begin{array}{l}\text { A união de aspectos sinérgicos da aprendizagem } \\
\text { experimental controlada em sala de aula e a } \\
\text { aplicação da aprendizagem em um projeto PBL } \\
\text { permite que os alunos aprendam mais facilmente } \\
\text { aplicar a teoria à prática, ajustando-se a } \\
\text { especificidades contextuais. }\end{array}$ \\
\hline 3 & $\begin{array}{l}\text { Desenvolver métodos de ensino LM } \\
\text { inovadores para estudantes de pós- } \\
\text { graduação com diferentes licenciaturas } \\
\text { (incluindo indivíduos sem experiência } \\
\text { industrial prévia), com base em três } \\
\text { estudos de caso baseados na indústria. }\end{array}$ & $\begin{array}{l}\text { Estudos de caso práticos } \\
\text { sobre a indústria. }\end{array}$ & $\begin{array}{l}\text { O curso de LM, ensinado do ponto de vista de um } \\
\text { engenheiro, enfatizou o papel do engenheiro na } \\
\text { fase inicial de concepção do produto e o projeto } \\
\text { do processo de fabricação, incluindo o projeto do } \\
\text { edifício. }\end{array}$ \\
\hline 4 & $\begin{array}{l}\text { Aplicar PBL a uma classe lean seis } \\
\text { sigmas para melhorar a capacidade } \\
\text { dos alunos ao aplicar conceitos como } \\
\text { parte de um exercício de consultoria, } \\
\text { preenchendo a lacuna analógica entre } \\
\text { a teoria, o caso e a prática real. }\end{array}$ & $\begin{array}{l}\text { Adicione a abordagem } \\
\text { PBL à classe em conjunto } \\
\text { com um projeto de } \\
\text { consultoria. }\end{array}$ & $\begin{array}{l}\text { Quando em conjunto com os exercícios práticos } \\
\text { na sala de aula, o trabalho de consultoria } \\
\text { proporcionou uma experiência de aprendizagem } \\
\text { positiva para os alunos e é recomendado para a } \\
\text { transferência eficaz de habilidades para a prática. }\end{array}$ \\
\hline 5 & $\begin{array}{l}\text { Propor um curso interdisciplinar } \\
\text { intitulado Princípios Lean e Aplicação, } \\
\text { que é tomado por alunos matriculados } \\
\text { em programas de graduação de } \\
\text { engenharia, negócios e } \\
\text { desenvolvimento de recursos humanos, } \\
\text { proporcionando-lhes o conhecimento e } \\
\text { habilidades para usar princípios lean e } \\
\text { ferramentas para resolver problemas } \\
\text { do mundo real. }\end{array}$ & $\begin{array}{l}\text { Projeto semestral: análise } \\
\text { do desempenho de uma } \\
\text { empresa local e } \\
\text { desenvolvimento de um } \\
\text { plano de implementação } \\
\text { de um programa lean. }\end{array}$ & $\begin{array}{l}\text { O desempenho das equipes dos alunos melhorou } \\
\text { conforme os projetos evoluíram. } \\
\text { Surpreendentemente, as soluções propostas se } \\
\text { concentraram principalmente em pequenas } \\
\text { atividades de melhoria contínua, ao invés de } \\
\text { recomendações para grandes redesenhos do } \\
\text { sistema de manufatura. }\end{array}$ \\
\hline 6 & $\begin{array}{l}\text { Aumentar as habilidades técnicas e } \\
\text { profissionais do aluno e o } \\
\text { conhecimento de negócios através da } \\
\text { integração da educação LM em um } \\
\text { programa de engenharia de fabricação } \\
\text { tradicional. }\end{array}$ & $\begin{array}{l}\text { Excursões em plantas, } \\
\text { palestras de convidados, } \\
\text { projetos industriais do } \\
\text { mundo real e simulação } \\
\text { lean Lego. }\end{array}$ & $\begin{array}{l}\text { Os resultados da avaliação interna dos cursos } \\
\text { feita pelos alunos no final do semestre indicam } \\
\text { que as abordagens complementares têm satisfeito } \\
\text { significativamente os alunos, cujos comentários } \\
\text { foram positivos. }\end{array}$ \\
\hline 7 & $\begin{array}{l}\text { Identificar as principais atividades de } \\
\text { simulação relacionadas com o ensino } \\
\text { de LM e avaliar a sua eficácia. }\end{array}$ & $\begin{array}{l}\text { PBL através de } \\
\text { simulações e jogos. }\end{array}$ & $\begin{array}{l}\text { Quatro lacunas são encontradas nos projetos de } \\
\text { simulação existentes: (i) falta de estresse sobre as } \\
\text { habilidades soft, (ii) um foco equivocado na "lean } \\
\text { linear", (iii) mal entendimento do papel-chave do } \\
\text { facilitador e (iv) falta de realismo. }\end{array}$ \\
\hline 8 & $\begin{array}{l}\text { Verificar como as técnicas e princípios } \\
\text { LM podem ser transferidos para } \\
\text { empresas e estudantes. }\end{array}$ & $\begin{array}{l}\text { Conferência, análise de } \\
\text { casos e estudo de } \\
\text { situações reais de } \\
\text { negócios. }\end{array}$ & $\begin{array}{l}\text { Vale a pena notar que os conceitos de LM são } \\
\text { transferíveis. As chaves para uma transferência } \\
\text { de LM bem-sucedida incluem treinamento da } \\
\text { força de trabalho, enquanto educa os alunos que } \\
\text { se tornarão os gerentes de operações no futuro. }\end{array}$ \\
\hline 9 & $\begin{array}{l}\text { Desenvolver um método de ensino LM } \\
\text { estruturado como uma palestra e uma } \\
\text { classe de discussão aberta. }\end{array}$ & $\begin{array}{l}\text { Palestras e simulações, } \\
\text { participação em grupos e } \\
\text { investigação de } \\
\text { aplicações industriais } \\
\text { reais. }\end{array}$ & $\begin{array}{l}\text { Embora o programa dependa dos alunos de pós- } \\
\text { graduação para representar o patrocinador da } \\
\text { empresa, este estilo, naturalmente, ainda viria } \\
\text { sem um programa de pós-graduação presente. A } \\
\text { exigência de ter um patrocinador da empresa } \\
\text { poderia ser cumprida através do uso de empresas } \\
\text { locais que estão dispostos a ter um registro de } \\
\text { funcionário para a classe e se tornar o "Campeão" } \\
\text { da equipe. As empresas patrocinadoras se } \\
\text { beneficiariam ao serem apresentadas à LM e ter } \\
\text { um projeto de equipe basicamente gratuito. }\end{array}$ \\
\hline 10 & $\begin{array}{l}\text { Avaliar e melhorar as competências } \\
\text { profissionais, abordando as múltiplas } \\
\text { necessidades das partes interessadas, } \\
\text { através de um único curso } \\
\text { experimental lean. }\end{array}$ & $\begin{array}{l}\text { Trabalhos práticos, visuais } \\
\text { e experienciais; trabalho } \\
\text { de projeto em empresas. }\end{array}$ & $\begin{array}{l}\text { As evidências devem ser claras quanto à } \\
\text { viabilidade e eficácia de incluir uma experiência } \\
\text { internacional em um curso de engenharia, é } \\
\text { possível impactar a competência global dos } \\
\text { alunos através de cursos no currículo de } \\
\text { engenharia. }\end{array}$ \\
\hline
\end{tabular}

Autores: (1) Johnson et al. (2003); (2) Conger and Miller (2013); (3) Peter (2010); (4) Conger and Miller (2014); (5) Van Til et al. (2005); (6) Fang et al. (2006); (7) Badurdeen et al. (2009); (8) Torres and Stephens (2005); (9) Lobaugh (2005); (10) Peters et al. (2008). 


\subsection{Dimensões dos estilos de aprendizagem}

Os estilos de aprendizagem são características cognitivas, afetivas e comportamentos psicológicos que servem como indicadores relativamente estáveis de como os alunos percebem, interagem e respondem ao ambiente de aprendizagem. Essencialmente, os estilos de aprendizagem são a forma como alguém tende a aprender melhor, compreendendo o método preferido de absorção, organização e sentido da informação (FELDER; BRENT, 2005; PUJ; AHMAD, 2016). Uma vez que diferentes pessoas processam a informação de diferentes maneiras, um estilo de aprendizagem não é nem preferível nem inferior a outro (CUEVAS, 2015).

No entanto, um paradigma prevalecente afirma que os estilos de aprendizagem podem ser obtidos a partir das experiências dos aprendizes no ambiente de aprendizagem em vez de serem uma propriedade inata de um indivíduo (KHATIBI; KHORMAEI, 2016; FRANCIS, 2016). Nesse sentido, evidências da literatura indicam que os métodos de ensino podem melhorar a aprendizagem do aluno quando correspondem à categoria de preferências de interação pré-existentes. Alternativamente, os estilos de aprendizagem podem ser alterados pela experiência com os métodos de ensino propostos (FELDER et al., 1998; KATSIOLOUDIS; FANTZ, 2012; RULTEGDE, 2016).

Pesquisas tem sido conduzidas através de décadas no que diz respeito às diferenças nos estilos de aprendizagem dos alunos, que são indicados como fator chave para explicar os diferentes resultados de vários métodos de ensino. Assim, o conceito de estilos de aprendizagem tem sido associado a uma grande diversidade de atributos dos alunos, implicando o desenvolvimento de vários modelos de estilos de aprendizagem (KALISKÁ, 2012). No entanto, não há consenso geral sobre os atributos de estilos de aprendizagem específicos (KHATIBI; KHORMAEI, 2016; ZHANG et al., 2016).

Por exemplo, no modelo de aprendizagem experiencial de Kolb (1984), os alunos são classificados de acordo com suas preferências por: (a) experiência concreta ou conceitualização abstrata e (b) experimentação ativa ou observação reflexiva. A combinação destas classificações resulta em quatro estilos de aprendizagem, nomeadamente: (I) divergente (concreto/reflexivo); (ii) assimilador (abstrato/reflexivo); (iii) convergente (abstrato/ativo); e (iv) acomodador 
(concreto/ativo). Mais tarde, Honey e Mumford (2000), baseados na teoria de Kolb, argumentaram que as principais características dos alunos podem ser integradas em quatro estilos de aprendizagem: ativo, reflexivo, teórico e pragmático. Além disso, muitos pesquisadores têm estudado extensivamente a relação entre os estilos de aprendizagem e outros aspectos individuais, como o efeito de interação da personalidade dos alunos (FELDER et al., 2002; PUJI; AHMAD, 2016) e a cultura e idade dos alunos (ARIANI, 2013; HUGHES, 2016).

Outro modelo amplamente reconhecido é o de Felder-Silverman (FELDER; SILVERMAN, 1988), que sugere a definição dos estilos de aprendizagem dos alunos de acordo com quatro dimensões: (I) percepção da informação, (ii) processamento da informação, (iii) entrada da informação e (iv) compreensão de informação. Para a percepção da informação, as preferências podem ser divididas em sensações (imagens visuais, sons, sensações físicas) e intuitivas (memórias, pensamentos, insights). Os sensores tendem a ser concretos e práticos, enquanto os intuitivos preferem abstrações, como modelos matemáticos e teorias (FELDER et al., 2002; KALISKÁ, 2012).

A segunda dimensão é categorizada de acordo com a forma como os alunos processam a informação: os alunos ativos são mais propensos a compreender os fatos através do engajamento em atividades físicas ou discussões; enquanto os alunos reflexivos são mais introspectivos e preferem pensar sobre eles em primeiro lugar. O input de informação é dividido principalmente em explicações verbais (explicações escritas ou faladas) e visuais (imagens, diagramas, demonstrações) (FELDER; SPURLIN, 2005).

Finalmente, a quarta dimensão compreende a forma como os alunos evoluem para a compreensão. Eles podem ser denotados como aprendizes sequenciais, que tendem a compreender melhor em uma progressão lógica de passos incrementais, ou aprendizes globais, que geralmente pensam de uma forma mais orientada para o sistema e preferem ver o "quadro geral" em primeiro lugar. O questionário ILS (FELDER; SOLOMAN, 2004) é um instrumento aplicado para avaliar essas quatro dimensões, indicando as preferências dos alunos de acordo com três níveis: (i) rasoavelmente bem equilibrado, (ii) preferência moderada e (iii) preferência muito forte. Em geral, uma vez que este modelo é bastante aceito entre os profissionais e acadêmicos, este estudo é baseado nas proposições deste modelo. 


\section{METODOLOGIA}

A metodologia deste trabalho examina a associação entre as preferências de aprendizagem de alunos e dois métodos de ensino LM, da seguinte forma: (I) métodos tradicionais, tais como palestras, exercícios em sala de aula e discussões de grupo e (ii) a abordagem PBL aplicada em problemas reais de empresas que passam por uma implementação lean. A Tabela 2 descreve cada método de ensino aplicado nas disciplinas de um curso de pós-graduação em Engenharia de Produção.

Além do método de ensino, vale a pena notar que o método avaliado para o desempenho dos alunos também variou de acordo com a disciplina, e os resultados de desempenho foram consolidados ao final da respectiva disciplina. Além disso, cada disciplina apresentava um objetivo e conteúdo de aprendizagem diferente, de modo que os conteúdos não se sobrepunham um ao outro permitindo uma compreensão mais ampla de LM. Estas disciplinas foram realizadas no primeiro e segundo trimestres de 2016, com duração de doze semanas cada. Os métodos de ensino foram utilizados indistintamente para expor e ensinar o conteúdo.

Tabela 2 - Métodos de ensino LM analisados

\begin{tabular}{|c|c|c|c|c|c|}
\hline No. & Descrição & Objetivo & Método de ensino & $\begin{array}{l}\text { Método de } \\
\text { avaliação }\end{array}$ & Sequência de conteúdos \\
\hline 1 & $\begin{array}{l}\text { LM - Práticas, } \\
\text { princípios e } \\
\text { conceitos. }\end{array}$ & $\begin{array}{l}\text { Compreensão } \\
\text { dos princípios e } \\
\text { conceitos de LM. }\end{array}$ & $\begin{array}{l}\text { Aulas teóricas; } \\
\text { Exercícios em equipe } \\
\text { e participação em } \\
\text { grupo; } \\
\text { Estudos de caso e } \\
\text { jogos curtos; } \\
\text { Passeios em plantas e } \\
\text { visitas em instalações } \\
\text { industriais. }\end{array}$ & $\begin{array}{l}\text { Participação na sala } \\
\text { de aula }(30 \%) ; \\
\text { Teste final }(70 \%) \text {. }\end{array}$ & $\begin{array}{l}\text { Introdução a LM; } \\
5 \text { S e gestão visual; } \\
\text { Mudança rápida; } \\
\text { Poka joke e jidoka; } \\
\text { Kanban e sistemas de puxar; } \\
\text { Trabalho padronizado; e } \\
\text { Manutenção produtiva total. }\end{array}$ \\
\hline 2 & $\begin{array}{l}\text { LM - PBL em } \\
\text { empresas em } \\
\text { fase de } \\
\text { implementação. }\end{array}$ & $\begin{array}{l}\text { Ligação de } \\
\text { conceitos e } \\
\text { princípios a } \\
\text { condições e } \\
\text { procedimentos } \\
\text { para aplicação de } \\
\text { LM. }\end{array}$ & $\begin{array}{l}\text { Aprendizagem } \\
\text { baseada em } \\
\text { problemas; } \\
\text { Treinamento e } \\
\text { orientação; } \\
\text { Atividades práticas. }\end{array}$ & $\begin{array}{l}\text { Apresentação do } \\
\text { relatório A3: } \\
\text { Professor (60\%); } \\
\text { Supervisor de equipe } \\
(40 \%) \text {. }\end{array}$ & $\begin{array}{l}\text { Explore o problema } \\
\text { apresentado; } \\
\text { Indique o que é conhecido; } \\
\text { Definir os problemas; } \\
\text { Pesquisa do conhecimento; } \\
\text { Investigar soluções; } \\
\text { Expor e apoiar a solução } \\
\text { escolhida; e } \\
\text { Rever o desempenho da } \\
\text { equipe. }\end{array}$ \\
\hline
\end{tabular}

\subsection{Características da amostra e coleta de dados}

A seleção da amostra de alunos incluiu apenas os alunos que cursaram ambas as disciplinas de LM do mesmo curso de pós-graduação em engenharia de produção, em uma das principais universidades públicas do sul do Brasil. O objetivo foi reduzir 
efeitos do ambiente externo (por exemplo, diferenças nos cursos de pós-graduação e ênfase na pesquisa de doutorado e mestrado, etc.), uma vez que isso seria relativamente homogêneo dentro da amostra. A escolha não aleatória dos respondentes que também já são conhecidos dos pesquisadores é uma estratégia comumente utilizada em outros estudos sobre LM (BOYLE et al., 2011; TORTORELLA et al., 2015). Por exemplo, Shah e Ward (2007) usaram uma amostra com participantes extraídos de cursos e eventos de treinamento quando realizaram uma pesquisa sobre LM, uma vez que era necessário que os entrevistados tivessem experiência no assunto.

As características dos quinze alunos incluídos na amostra do estudo são apresentadas na Tabela 3. A amostra estudada apresenta uma quantidade desequilibrada de alunos de doutorado e mestrado $(26,67 \%$ e $73,33 \%$, respectivamente). Além disso, 33,33\% dos alunos alegaram ter experiência prévia com princípios e técnicas de LM; 46,67\% deles têm bolsa de estudos, dedicando seu tempo integral aos seus cursos de pós-graduação; $40 \%$ tinham menos de trinta anos; e $60 \%$ eram do sexo masculino.

Tabela 3 - Características dos alunos

\begin{tabular}{ccccccccc}
\hline & \multicolumn{2}{c}{$\begin{array}{c}\text { Experiência } \\
\text { prévia com LM? }\end{array}$} & \multicolumn{2}{c}{$\begin{array}{c}\text { Dedicação do } \\
\text { aluno }\end{array}$} & \multicolumn{2}{c}{ Idade } & \multicolumn{2}{c}{ Gênero } \\
\hline Grau de & Não & Sim & Tempo & Tempo & $<30$ & $>30$ & Masculi & Feminin \\
aplicação & & & integral & parcial & anos & anos & no & 0 \\
Doutor & 1 & 3 & 2 & 2 & 1 & 3 & 2 & 2 \\
Mestre & 9 & 2 & 5 & 6 & 5 & 6 & 7 & 4 \\
Total & 10 & 5 & 7 & 8 & 6 & 9 & 9 & 6 \\
\hline
\end{tabular}

Em relação aos estilos de aprendizagem, adaptamos e aplicamos o questionário ILS com os alunos de pós-graduação para verificar o estilo de aprendizagem preferido (FELDER; SOLOMAN, 20 04; FELDER; SPURLIN 2005). Os estilos de aprendizagem são formas preferidas de organizar o que se vê, se lembra e pensa sobre um assunto. Assim, o perfil de estilo de aprendizagem de um aluno fornece uma indicação de forças prováveis e possíveis tendências ou hábitos que podem levar a dificuldades em ambientes acadêmicos (FELDER; SILVERMAN, 1988; KALISKÁ, 2012).

O ILS é um instrumento constituído por 44 questões utilizadas para avaliar as preferências em quatro dimensões de um estilo de aprendizagem: (i) processamento 
da informação (ativo/reflexivo), (ii) percepção da informação (sensorial/intuitivo), (iii) entrada da informação (visual/verbal), e (iv) compreensão da informação (sequencial/global). Os resultados do questionário simplesmente indicam preferências para cada dimensão, e as sugestões que acompanham os resultados podem permitir verificar a correspondência com o método de ensino (FELDER et al., 1998, LITZINGER et al., 2007). A avaliação dos estilos de aprendizagem foi realizada em ambas as disciplinas para verificar inconsistências. Os alunos preencheram um questionário online (FELDER; SOLOMAN, 2016) que fornece automaticamente o resultado das preferências para cada dimensão. Não foram encontradas nenhuma inconsistência ou mudança no estilo de aprendizagem de nenhum dos alunos analisados.

\subsection{Análise de dados}

A análise dos dados foi realizada utilizando o software estatístico SPSS ${ }^{\circledR}$ versão 24. As 15 respostas foram testadas de acordo com o efeito das dimensões dos estilos de aprendizagem em cada método de ensino. Em primeiro lugar, foi aplicada uma análise de variância para os grupos (One-Way ANOVA) para identificar o efeito principal significativo entre as dimensões do estilo de aprendizagem para cada método de ensino. Se os grupos são formados por mais de uma dimensão, o efeito pode ser atribuível à interação entre as dimensões. $\mathrm{Na}$ análise da interação entre duas variáveis independentes, recomenda-se a utilização de um desenho fatorial duplo (Two-Way ANOVA) (TABACHNICK; FIDELL, 2013; MONTGOMERY, 2013).

A hipótese alternativa da análise Two-Way ANOVA testa a presença de efeito de interação significativo entre os fatores analisados. Uma análise de variância TwoWay foi aplicada para identificar interações significativas entre as dimensões do estilo de aprendizagem para cada método de ensino. O uso de ANOVA One e Two-Way requer a suposição de que os erros do modelo são distribuídos de forma normal e independente (MEYERS et al., 2006). Realizou-se para ambos os modelos uma análise visual dos resíduos e o teste de Kolmogorov-Smirnov para a sua adesão à distribuição normal. Todos os pré-requisitos foram satisfeitos para realizar o teste ANOVA. 
Portanto, quando houve um efeito principal significativo e também um efeito de interação significativo no Modelo Fatorial Two-Way, o efeito principal dos fatores envolvidos na interação foram desconsiderados (MONTGOMERY, 2013). Vale ressaltar que não há tamanho mínimo de amostra para o teste ANOVA, pois os resultados significativos são influenciados pela magnitude das diferenças entre os dois grupos (MEYERS et al., 2006). No entanto, um modelo fatorial completo não foi testado porque havia um baixo número de respostas para fornecer interações de ordem superior. Todos os testes foram realizados com um nível de significância (alfa) de 0,10 . As interações que apresentaram um efeito significativo aceitaram o tamanho da amostra com os respectivos graus de liberdade necessários.

\section{RESULTADOS}

A Tabela 4 apresenta os resultados para o efeito principal de cada dimensão do estilo de aprendizagem no método de ensino correspondente. Para a primeira disciplina, que é ensinada com base nos métodos de ensino tradicionais, nenhuma dimensão apresenta efeito principal significativo quando analisada individualmente. Este resultado é um tanto surpreendente à luz da sabedoria convencional que sugere que os métodos tradicionais de ensino, tais como palestras e discussões em sala de aula, podem ajudar os alunos a compreender melhor os conceitos e princípios de um determinado assunto, especialmente quando eles têm pouca experiência sobre ele (GIJBLES et al., 2005). Os métodos tradicionais de ensino têm sido amplamente utilizados na educação de engenharia e, apesar de sua ampla aplicação, nossos resultados mostram que esses métodos não estão associados a qualquer preferência de aprendizagem particular para LM aprendizagem.

Tabela 4 - Efeitos principais das dimensões do estilo de aprendizagem para os métodos de ensino LM propostos

\begin{tabular}{|c|c|c|}
\hline Dimensões do estilo de aprendizagem & $\begin{array}{c}\text { Métodos } \\
\text { tradicionais }\end{array}$ & Método PBL \\
\hline Processamento da informação & - & $\mathrm{SD}^{*}$ \\
\hline Percepção da informação & - & $S D^{* *}$ \\
\hline Entrada da informação & - & - \\
\hline Compreensão da informação & - & - \\
\hline
\end{tabular}


Por outro lado, para a abordagem PBL aplicada na segunda disciplina, as dimensões 'processamento da informação' e 'percepção da informação' parecem estar significativamente associadas a este método de ensino. Constatou-se um crescente interesse em usar PBL no aprendizado de LM, o que está alinhado com os esforços atuais para passar da apresentação descontextualizada de conteúdo técnico (normalmente exibida nos métodos de ensino tradicionais) à integração holística de conteúdo e prática (GALAND et al., 2012; JONES et al., 2013). Entretanto, como os resultados da Two-Way ANOVA mostraram que o processamento da informação e a percepção da informação apresentam um efeito de interação significativo com a entrada de informação (Tabela 5), os efeitos principais foram desconsiderados e o foco da discussão ficou nas interações.

Tabela 5 - Efeitos de interação das dimensões do estilo de aprendizagem para os métodos de ensino LM propostos

\begin{tabular}{lcc}
\hline \multicolumn{1}{c}{ Interações } & $\begin{array}{c}\text { Métodos } \\
\text { tradicionais }\end{array}$ & Método PBL \\
\hline Processamento * Percepção & $\mathrm{SD}^{*}$ & - \\
Processamento * Entrada & - & $\mathrm{SD}^{*}$ \\
Processamento * Compreensão & - & - \\
Percepção * Entrada & - & $\mathrm{SD}^{*}$ \\
Percepção * Compreensão & - & - \\
Entrada * Compreensão & - & - \\
\hline *Diferença significativa a 5\% & &
\end{tabular}

A análise dos efeitos das interações foi realizada comparando duas dimensões de aprendizagem ao mesmo tempo em cada método de ensino. Para os métodos tradicionais, a interação entre o processamento da informação e a percepção da informação mostrou-se significativa ( $p$-valor $<0,10$ ). Este resultado indica que 0 desempenho dos alunos nesta disciplina depende da combinação das preferências de aprendizagem nessas duas dimensões. A Figura 1 mostra como o desempenho dos alunos varia de acordo com as combinações correspondentes. Os alunos que são mais propensos a reter as informações por discuti-las ou aplicá-las (alunos ativos) e preferem aprender através da resolução de problemas com métodos bem estabelecidos (sensores), mostram um melhor desempenho com os métodos tradicionais de ensino LM.

Em oposição, os alunos ativos que frequentemente preferem descobrir possibilidades e relacionamentos e não gostam de repetição demonstram níveis mais 
baixos de compreensão do LM se os métodos tradicionais são empregados. Quando os alunos preferem pensar sobre o assunto e trabalhar sozinhos (alunos reflexivos), a maneira como eles percebem a informação (intuitiva ou sensorial) não mostra ser significativa. Em geral, a Tabela 6 mostra os meios marginais estimados para a interação entre o processamento da informação e a percepção da informação. De fato, assumindo um intervalo de confiança de $90 \%$, os métodos tradicionais de ensino LM fornecem diferenças significativas no desempenho dos alunos quando comparados os alunos que são intuitivos ativos e sensores ativos. Nenhuma diferença significativa no desempenho dos alunos pode ser inferida ao comparar intuitivos reflexivos, sensores reflexivos e intuitivos ativos.

Este resultado corrobora os achados de Conger e Miller $(2013,2014)$ e Van Til et al. (2005), que sugerem que a utilização única dos métodos tradicionais de ensino e aprendizagem de LM não são tão eficazes para uma ampla gama de estudantes com diferentes contextos teóricos. Além disso, particularmente para o ensino de LM, pesquisadores como Seddon e Caulkin, (2007) pediram a integração de técnicas de aprendizagem de ação, para que os alunos se tornem mais engajados e tenham a oportunidade de efetivamente desenvolver seus conhecimentos de acordo com as especificidades do contexto. No entanto, essas técnicas não são facilmente adaptadas ou efetivamente integradas em disciplinas cujo método de ensino é centrado em palestras e exercícios em sala de aula (HALL; HOLLOWAY, 2008).

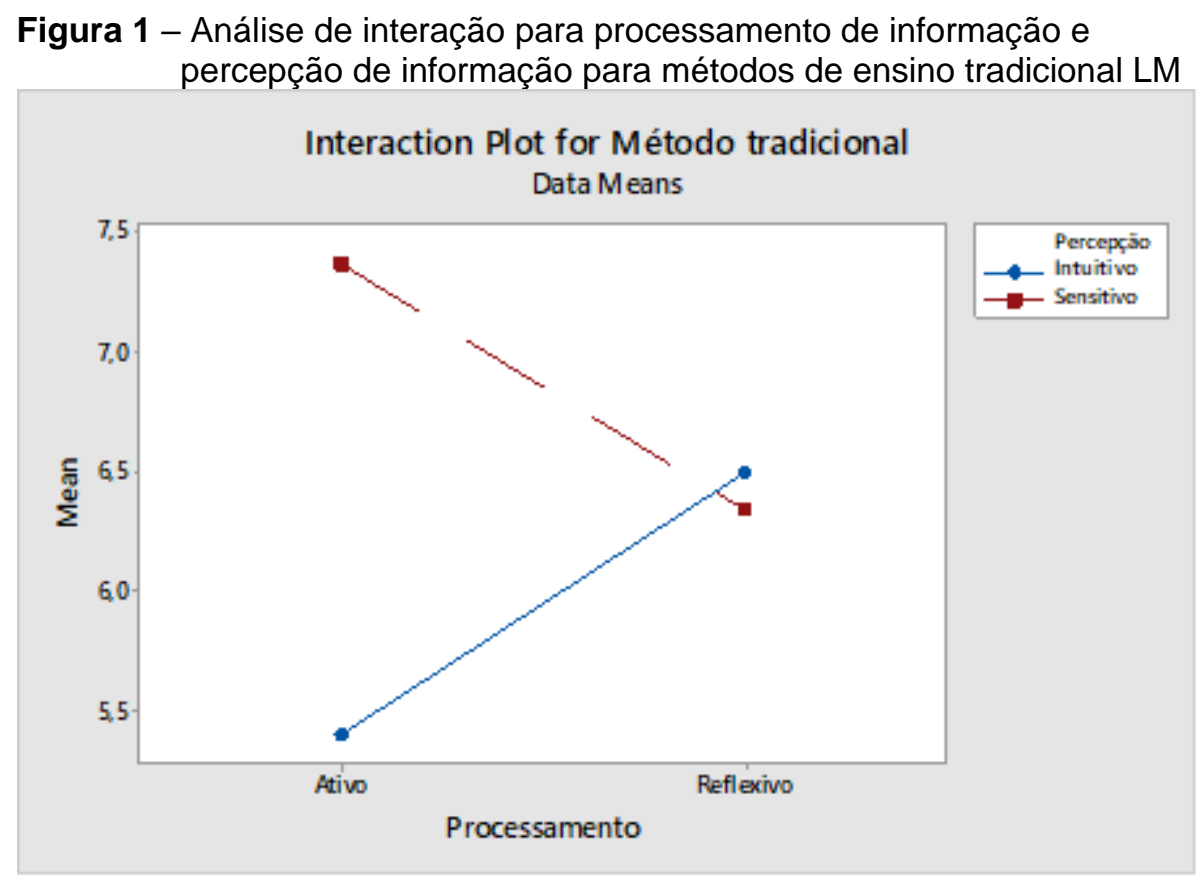


Tabela 6 - Médias marginais estimadas para interação do processamento da informação e da percepção da informação

\begin{tabular}{llcccc}
\hline $\begin{array}{l}\text { Processament } \\
\text { o }\end{array}$ & Percepção & Média & Limite inferior & $\begin{array}{c}\text { Limite } \\
\text { superior }\end{array}$ & Intervalo de confiança 90\% \\
\hline \multirow{2}{*}{ Reflexivo } & Intuitivo & 6.500 & 5.659 & 7.341 & \\
& Sensitivo & 6.340 & 5.588 & 7.092 & \\
\multirow{2}{*}{ Ativo } & Intuitivo & 5.400 & 4.429 & 6.371 & \\
& Sensitivo & 7.367 & 6.395 & 8.338 & $\ldots$ \\
\hline
\end{tabular}

Com relação à abordagem PBL, a Figura 2 mostra a variação de desempenho dos alunos de acordo com as preferências de aprendizagem combinadas para entrada de informação e processamento de informação. Além disso, a Tabela 7 apresenta os resultados para a média marginal estimada para esta interação considerando um intervalo de confiança de $90 \%$. Esses resultados indicam que a melhora na capacidade dos alunos de entender e aplicar os princípios e técnicas de LM não é significativamente diferente quando comparados os alunos ativos (tanto visuais como verbais) e os alunos reflexivos com preferência visual.

$\mathrm{Na}$ verdade, apenas alunos verbais que processam ativamente a informação mostraram entender melhor e aplicar os princípios e práticas LM do que os alunos que preferem a mesma informação, mas são mais reflexivos. Em oposição, o desempenho de alunos visuais, que lembram melhor do que veem, não mostra ser afetado pela forma como a informação é processada. Nos cursos de LM que aplicam PBL, os alunos são encorajados a trabalhar em equipe para resolver problemas complexos e autênticos que possibilitam o desenvolvimento de conhecimentos de conteúdo e capacidade de resolução de problemas, raciocínio, comunicação e auto-avaliação (BÉDARD et al., 2012; TOVAR; WARSHAWSKY, 2015). Portanto, é razoável esperar que, sob este tipo de método de ensino, os alunos ativos sejam mais propensos a um melhor desempenho do que os alunos reflexivos, mesmo que eles apresentem a mesma preferência de entrada de informações.

Para a interação entre entrada de informação e percepção de informação, a Figura 3 apresenta os desempenhos dos alunos de acordo com os estilos combinados, enquanto a Tabela 8 mostra a média marginal estimada com intervalo de confiança de $90 \%$. O desempenho na disciplina PBL de alunos visuais não mostrou ser significativamente influenciado pela maneira como os alunos percebem a informação (intuitiva ou sensorial). Além disso, não foi encontrada diferença 
significativa para o desempenho de intuitivos verbais e intuitivos visuais; O mesmo é observado para a comparação entre intuitivos visuais e sensores verbais.

No entanto, os resultados deste método de ensino indicam que o desempenho dos sensores, independentemente de suas preferências de entrada de informação (visual ou verbal), tende a ser maior do que os intuitivos verbais. O PBL é caracterizado como uma abordagem centrada no estudante, em que os professores são facilitadores em vez de disseminadores, e os problemas abertos servem como motivação inicial e estrutura para o aprendizado (BARRON et al., 1998). Hitt (2010) complementa que as atividades realizadas em uma abordagem PBL devem ser aquelas valorizadas no mundo real, e uma análise e discussão sobre o que foi aprendido com esse problema são essenciais.

Assim, a conexão entre as necessidades de aprendizagem e os problemas do mundo real evidenciados por fatos tangíveis pode soar atraente para sensores, justificando seu maior desempenho nesta disciplina. Por outro lado, uma vez que os problemas são projetados para serem complexos, reflexivos de práticas realistas e enquadrados para promover a aquisição de conhecimento, resolução de problemas, aprendizagem autodirigida e interesse (HMELO-SILVER, 2004; SANTOS; SILVA, 2015), os intuitivos visuais podem se beneficiar desta abordagem, explicando porque nenhuma diferença significativa foi encontrada entre esta combinação de estilo e sensores verbais/visuais.

Figura 2 - Análise de interação para entrada de informação e processamento de informação para abordagem PBL

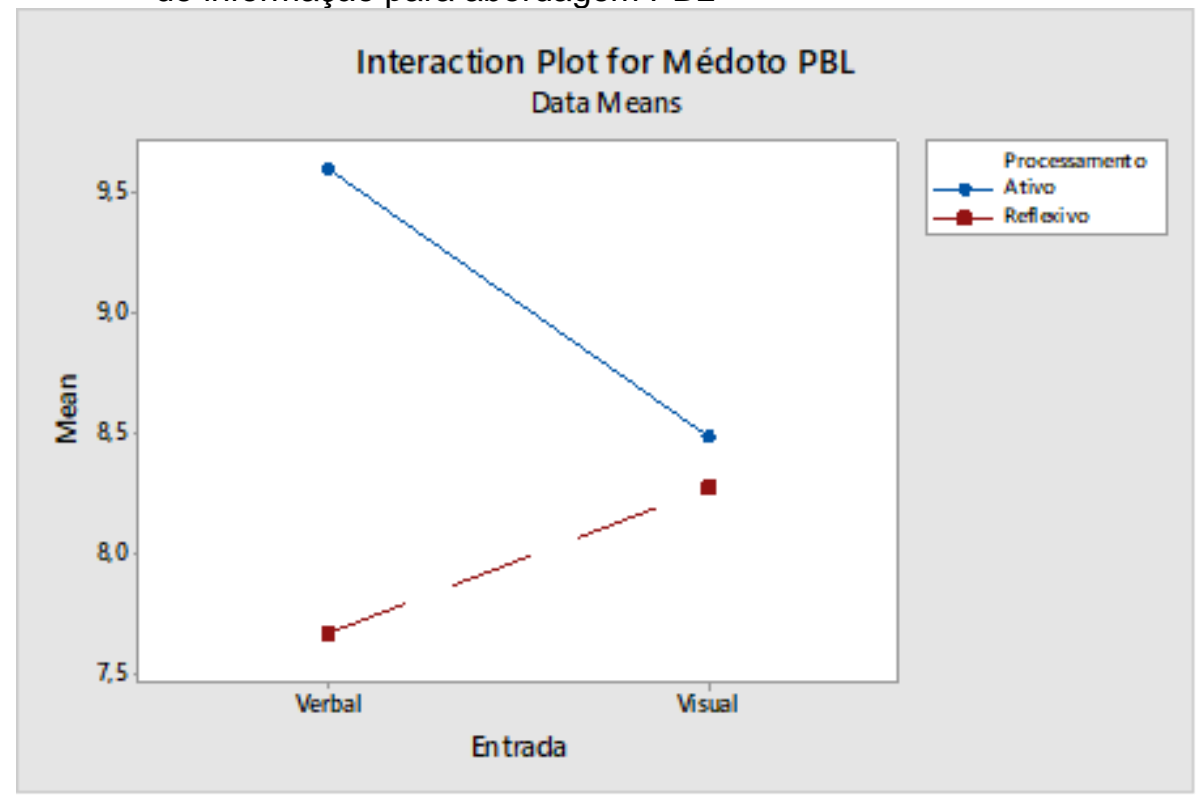

Revista Produção Online. Florianópolis, SC, v. 18, n. 1, p. 93-117, 2018. 
Tabela 7 - Médias marginais estimadas para a interação do processamento da informação e da entrada de informação

\begin{tabular}{llcccc}
\hline Processamento & Entrada & Média & Limite inferior & $\begin{array}{c}\text { Limite } \\
\text { superior }\end{array}$ & Intervalo de confiança 90\% \\
\hline \multirow{2}{*}{ Reflexivo } & Verbal & 7.667 & 7.039 & 8.294 & \\
\multirow{2}{*}{ Ativo } & Visual & 8.267 & 7.823 & 8.710 & \\
& Verbal & 9.600 & 8.513 & 10.687 & $\ldots$ \\
\hline
\end{tabular}

Figura 3 - Análise de interação para entrada de informação e percepção de informação para abordagem PBL

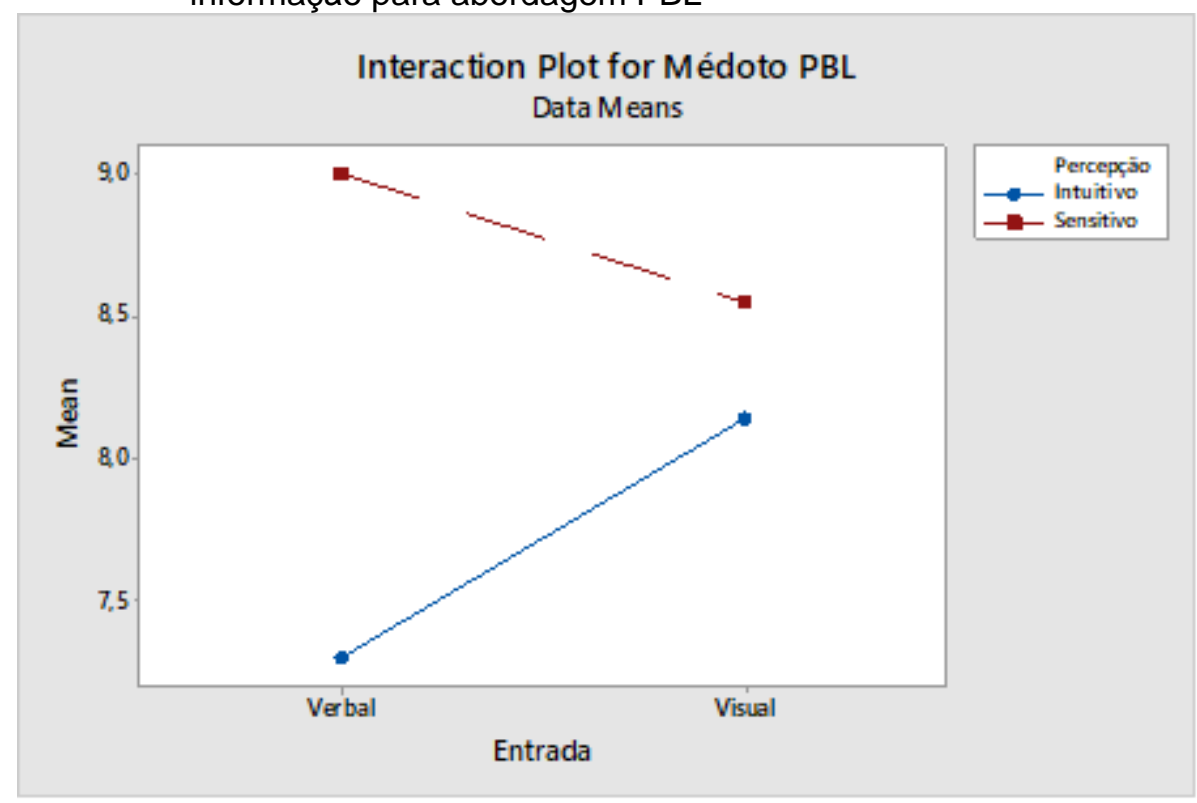

Tabela 8 - Médias marginais estimadas para a interação da percepção da informação e da entrada de informação

\begin{tabular}{llcccc}
\hline Percepção & Entrada & Média & Limite inferior & $\begin{array}{c}\text { Limite } \\
\text { superior }\end{array}$ & Intervalo de confiança 90\% \\
\hline \multirow{2}{*}{ Intuitivo } & Verbal & 7.300 & 6.573 & 8.027 & - \\
& Visual & 8.140 & 7.680 & 8.600 & $-\ldots$ \\
\multirow{2}{*}{ Sensitivo } & Verbal & 9.000 & 8.273 & 9.727 & \\
& Visual & 8.550 & 8.130 & 8.970 & \\
\hline
\end{tabular}

Surpreendentemente, nenhum dos métodos de ensino de LM propostos apresentou uma associação significativa (efeito principal ou efeito de interação) com a dimensão da compreensão da informação. Uma vez que os conteúdos no método de ensino tradicional foram apresentados em uma abordagem passo a passo em sala de aula, os alunos sequenciais, que tendem a ganhar entendimento de forma linear e evolutiva, deveriam se beneficiar desse método. Os resultados obtidos para a amostra do estudo não evidenciam a existência dessa relação, indicando que ela parece ser menos invasiva do que o esperado. 
Em oposição, a disciplina PBL foi projetada de modo que os alunos globais, que são mais propensos a resolver problemas complexos rapidamente, devem ter um melhor desempenho. Os resultados não suportam tal suposição. No entanto, Hong (2007) ressalta que a adaptação dessa abordagem a uma estrutura de curso existente poderia, ocasionalmente, alterar os efeitos de suas raízes originais nas escolas de medicina.

\section{CONCLUSÕES}

Neste artigo, investigou-se a relação entre estilos de aprendizagem e métodos de ensino para melhorar a aprendizagem de LM de estudantes de engenharia de produção de um curso de pós-graduação. As implicações deste estudo são de grande importância e relevância tanto para pesquisadores como para profissionais.

Esta pesquisa sugere dois achados principais. Em primeiro lugar, a pesquisa apresenta algumas contribuições teóricas importantes para o estado-da-arte sobre ensino e aprendizagem de LM. É proposta uma nova abordagem para identificar a relação entre os estilos de aprendizagem dos alunos e os métodos de ensino habituais que podem contribuir para uma compreensão bem-sucedida dos princípios e técnicas de LM. A literatura especializada sobre ensino e aprendizagem de LM frequentemente afirma que professores e instrutores adotam principalmente um único método de ensino, tais como palestras e discussões em sala de aula, negligenciando o fato de que uma aprendizagem efetiva é governada tanto pelas habilidades nativas dos alunos quanto pela correta relação entre os atributos dos alunos, como aprendizes, e o estilo de ensino do professor.

A abordagem identifica estilos de aprendizagem preferidos que podem contribuir para os métodos de ensino LM, proporcionando maior desempenho dos alunos nas disciplinas. Isso é realizado através de uma análise Two-Way ANOVA aplicada com quinze estudantes de pós-graduação que cursaram duas disciplinas LM com diferentes abordagens de ensino. Usando essa proposição, os acadêmicos podem escolher o método de ensino LM com maior probabilidade de contribuir para os estilos de aprendizagem preferidos dos alunos. Vale notar que, apesar de ser impossível adaptar o método de ensino de acordo com o estilo de aprendizagem de 
cada aluno em uma classe, é igualmente equivocado supor que um único método de ensino pode satisfazer as necessidades de cada aluno.

Particularmente para a educação LM, os resultados fornecem evidências para apoiar a aplicação de um método de ensino híbrido, em que a primeira parte do curso é ensinado em um cenário tradicional, para definir os conceitos fundamentais, seguido de um período culminante que oferece uma oportunidade para estender os conhecimentos adquiridos em problemas reais e práticos. Portanto, a abordagem educacional apropriada para o ensino e aprendizagem de LM ajudaria a aumentar a capacidade dos alunos para adquirir e aplicar o conhecimento em situações do mundo real, preparando-os para atender as competências necessárias que atendam às demandas atuais das organizações ou da academia.

Em segundo lugar, apesar da integração de métodos de ensino complementares ser necessária para uma aprendizagem eficaz de LM, nem todos os estudantes de pós-graduação beneficiam-se na mesma medida. As preferências de aprendizagem dos alunos parecem influenciar a eficácia dos métodos de ensino e, portanto, devem ser levados em conta ao projetar o curso de LM. No entanto, estas preferências não são facilmente identificadas a priori, minando a assertividade dos métodos de ensino. Tal fato denota a importância de compreender o perfil dos futuros alunos para que os programas e cursos de pós-graduação possam se adaptar adequadamente. Portanto, a iniciativa isolada de incorporar um método de ensino específico nos cursos de LM não garante uma melhoria no processo de aprendizagem dos alunos de pós-graduação. De fato, os resultados indicam que as preferências de aprendizagem dos alunos são um elemento-chave para a concepção dos métodos de ensino e devem ser sistematicamente seguidas e utilizadas pelos programas de pósgraduação em engenharia de produção, a fim de proporcionar melhores resultados de aprendizagem e assertividade pedagógica.

Existem algumas limitações devido à natureza da amostra utilizada na pesquisa que deve ser destacada. Primeiro, o tamanho reduzido da amostra estudada prejudica o teste de um modelo fatorial completo que verifica as interações de ordem superior. Portanto, a análise de dados dos efeitos das interações é limitada a comparações entre duas dimensões de aprendizagem de cada vez para cada método de ensino. Além disso, no que diz respeito à relação entre o ILS e os métodos de ensino LM, pesquisas futuras são necessárias para expandir a investigação para outros métodos 
de ensino além do PBL e métodos tradicionais. Assim, uma melhor compreensão da influência simultânea de vários métodos de ensino e as preferências de aprendizagem dos alunos pode fornecer argumentos aos professores e acadêmicos para antecipar seu efeito sinérgico, de modo que os resultados finais são mais propensos a satisfazer as expectativas.

Finalmente, uma vez que o estudo foi realizado com comparações das preferências de aprendizagem dos alunos e seus desempenhos dentro de cada método de ensino, uma investigação mais aprofundada poderia averiguar uma possível diferença de performance entre os métodos de ensino. Portanto, não só a associação entre os estilos de aprendizagem e os métodos de ensino estariam em análise, mas também os desempenhos dos alunos entre os métodos de ensino.

\section{REFERÊNCIAS}

ALVES, A.; DINIS-CARVALHO, J.; SOUSA, R. Lean production as promoter of thinkers to achieve companies' agility. Learning Organization, v. 19, n. 3, p. 219-237, 2012. https://doi.org/10.1108/09696471211219930

ALVES, A.; KAHLEN, F.; FLUMERFELT, S.; MANALANG, A. The lean production multidisciplinary: from operations to education. $7^{\text {th }}$ International Conference on Production Research, Americas, 2014. http://hdl.handle.net/1822/36785

ARIANI, D. Personality and learning motivation. European Journal of Business and Management, v. 5, p. 10-26, 2013.

BADURDEEN, F.; MARKSBERRY, P.; HALL, A.; GREGORY, B. Teaching lean manufacturing with simulations and games: a survey and future directions. Simulation \& Gaming, 2009. https://doi.org/10.1177/1046878109334331

BARRON, B.; SCHWARTZ, D.; VYE, N.; MOORE, A.; PETROSINO, A.; ZECH, L.; BRANSFORD, J. Doing with understanding: lessons from research on problem- and project-based learning. The Journal of the Learning Sciences, v.7, n. 3-4, p. 271-311, 1998. http://dx.doi.org/10.1080/10508406.1998.9672056

BAYRAM, S.; DENIZ, L.; ERDOĞAN, Y. The role of personality traits in web-based education. The Turkish Online Journal of Educational Technology-TOJET, v.7, n. 2, p. 5-41, 2008.

BÉDARD, D.; LISON, C.; DALLE, D.; CÔTÉ, D.; BOUTIN, N. Problem-based and projectbased learning in engineering and medicine: determinants of students' engagement and persistence. Interdisciplinary Journal of Problem-Based Learning, v. 6, n. 2, p. 7-30, 2012.

BEDDOES, K.; JESIEK, B.; BORREGO, M. Identifying opportunities for collaboration in international engineering education research on problem- and project-based learning. 
Interdisciplinary Journal of Problem-Based Learning, v. 6, n. 2, p. 7-34, 2010. https://doi.org/10.7771/1541-5015.1142

BIGGS, J. Teaching for quality learning at university. The society for research into higher education and open university press, Buckingham, 2003.

BOYLE, T.; SCHERRER-RATHJE, M.; STUART, I. Learning to be lean: the influence of external information sources in lean improvements. Journal of Manufacturing Technology Management, v. 22, n. 5, p. 587-603, 2011. https://doi.org/10.1108/17410381111134455

CAMPOS, L.; LIMA, R.; ALVES, A.; MESQUITA, D.; MOREIRA, F.; CAMPOS, B. Fatores críticos num processo de aprendizagem baseada em projetos: percepções de estudantes de $1^{\circ}$ ano de engenharia, Pontifical Catholic University of Sao Paulo, Brazil, 2013. Disponível em: https://repositorium.sdum.uminho.pt/handle/1822/30290. Acesso em: 27 julho 2017.

CARVALHO, C.; LOPES, M.; ANTÓNIO, G.; ÁVILA, P.; BASTOS, J.; FONSECA, L. Lean learning academy: na innovative framework for lean manufacturing training. $1^{\text {st }}$ International Conference of the Portuguese Society for Engineering Education (CISPEE), p. 0-4, 2013. https://doi.org/10.1109/CISPEE.2013.6701958

CONGER, S.; MILLER, R. Problem-based learning for a lean six sigma course. University of Dallas, USA. Sprouts: Working Papers on Information Systems, v.13, n. 1, 2013.

CONGER, S.; MILLER, R. Problem-based learning applied to student consulting in a lean production course. Journal of Higher Education Theory and Practice, v. 14, n. 1, p. 81, 2014.

CUEVAS, J. Is learning styles-based instruction effective? A comprehensive analysis of recent research on learning styles. Theory and Research in Education, v. 13, n. 3, p. 308-333, 2015. https://doi.org/10.1177/1477878515606621

FANG, N.; COOK, R.; HAUSER, K. Work in progress: an innovative interdisciplinary lean manufacturing course. Frontiers in Education Conference, $36^{\text {th }}$ Annual Conference, IEEE, p. 13-14, 2006. https://doi.org/10.1109/FIE.2006.322447

FELDER, R.; BRENT, R. Understanding student differences. Journal of Engineering Education, v. 94, n. 1, p. 57-72, 2005. https://doi.org/10.1002/j.2168-9830.2005.tb00829.x

FELDER, R.; FELDER, G.; DIEZ, E. A longitudinal study of engineering student performance and retention versus comparisons with traditionally-taught students. Journal of Engineering Education, v. 87, n. 4, p. 469-480, 1998. http://dx.doi.org/10.1002/j.21689830.1998.tb00381.x

FELDER, R.; FELDER, G.; DIETZ, E. The effects of personality type on engineering student performance and attitudes. Journal of Engineering Education, v. 91, n. 1, p. 317, 2002. http://dx.doi.org/10.1002/j.2168-9830.2002.tb00667.x

FELDER, R.; SILVERMAN, L. Learning and teaching styles in engineering education. Engineering Education, v. 78, n. 7, p. 674-681, 1998. 
FELDER, R.; SOLOMAN, B. Index of Learning Styles (ILS). Disponível em: $<$ www2.ncsu.edu/unity/lockers/users/f/felder/public/lLSpage.html>. Acesso em: 5 maio 2016.

FELDER, R.; SPURLIN, J. Applications, reliability and validity of the index of learning styles. International Journal of Engineering Education, v. 21, n. 1, p. 103-112, 2005. Disponível em: https://wss.apan.org/jko/mls/Learning\%20Content/lLS Validation(IJEE).pdf. Acesso em: 27 julho 2017.

FLIEDNER, G.; MATHIESON, K. Learning lean: a survey of industry lean needs. Journal of Education for Business, v. 84, n. 4, p. 194-199, 2009. http://dx.doi.org/10.3200/JOEB.84.4.194-199

FLUMERFELT, S.; KAHLEN, F.; ALVES, A.; SIRIBAN-MANALANG, A. Lean Engineering Education: content and competency mastery, ASME Press, New York, 2014.

FRANCIS, R. Learning styles: key to enhance learning among student teachers of the B. ED course. International Education and Research Journal, v. 2, n. 12, p. 54-55, 2016. Disponível em: http://ierj.in/journal/index.php/ierj/article/view/592. Acesso em: 27 julho 2017.

GALAND, B.; FRENAY, M.; RAUCENT, B. Effectiveness of problem-based learning in engineering education: a comparative study on three levels of knowledge structure. International Journal of Engineering Education, v. 28, n. 4, p. 939-947, 2012.

GIJBLES, D.; DOCHY, F.; VAN DEN BOSSCHE, P.; SEGERS, M. Effects of problembased learning: a meta-analysis from the angle of assessment. Review of Educational Research, v. 75, n. 1, p. 27-61, 2005. https://doi.org/10.3102/00346543075001027

HALL, A.; HOLLOWAY, L. Application of lean concepts to the teaching of lean manufacturing. 2007 American Society for Engineering Education Annual Conference \& Exposition, 2008.

HITT, J. Problem-based learning in engineering. Center for teaching excellence, United States Military Academy, West Point, NY, 2010.

HMELO-SILVER, C. Problem-based learning: what and how do students learn? Educational Psychology Review, v.16, n. 3, p. 235-266, 2004. https://doi.org/10.1023/B:EDPR.0000034022.16470.f3

HONEY, P.; MUMFORD, A. The learning styles helper's guide. Primeira publicação em set. 2000, edição revisada em apr. 2006, Disponível em: <www.peterhoney.com>.

HONG, J. The comparison of problem-based learning (PmBL) model and project-based learning (PtBL) model. International Conference on Engineering Education (ICEE) 2007, Coimbra, Portugal, 2007. Disponível em: <

http://www.ineer.org/Events/ICEE2007/papers/179.pdf.>. Acesso em: 27 julho 2017.

HUGHES, G. Identifying learning styles: A CPD article improved Grace Hughes's knowledge of how to identify different learning styles. Nursing Standard, v. 31, n. 16-18, p. 72-73, 2016. https://doi.org/10.7748/ns.31.16-18.72.s49

JONES, B.; EPLER, C.; MOKRI, P.; BRYANT, L.; PARETTI, M. The effects of a collaborative problem-based learning experience on students' motivation in engineering 
capstone courses. Interdisciplinary Journal of Problem-Based Learning, v.7, n 2, p. 3471, 2013. https://doi.org/10.7771/1541-5015.1344

JOHNSON, S.; GERSTENFELD, A.; ZENG, A.; RAMOS, B.; MISHRA, S. Teaching lean process design using a discovery approach. American Society for Engineering Education Annual Conference \& Exposition, USA, 2003.

KALISKÁ, L. Felder's Learning Style Concept and its Index of Learning Style Questionnaire in the Slovak Conditions. Grant Journal, v.1, p. 52-56, 2012. Disponível em: http://www.grantjournal.com/issue/0101/PDF/0101kaliska.pdf. Acesso em: 27 julho 2017.

KANIGOLLA, D.; CUDNEY, E.; CORNS, S. Enhancing engineering education using project-based learning for lean and six sigma. International Journal of Lean Six Sigma, v. 5, n.1, p. 45-61, 2014. https://doi.org/10.1108/lJLSS-02-2013-0008

KATSIOLOUDIS, P.; FANTZ, T. A comparative analysis of preferred learning and teaching styles for engineering, industrial, and technology education: students and faculty. Journal of Technology Education, v. 23, n. 2, p. 61-69, 2012.

https://doi.org/10.21061/jte.v23i2.a.4

KETTERING UNIVERSITY. Lean resource center, Disponível em:

$<$ https://paws.kettering.edu/ wschelle/Ford Grad/Ford Ket.htm>, Acesso em: 20 dez. 2016.

KHATIBI, M.; KHORMAEI, F. Learning and personality: a review. Journal of Educational and Management Studies, v. 6, n. 4, p. 89-97, 2016. Disponível em: < http://jems.scienceline.com/attachments/article/40/J.\%20Educ.\%20Manage.\%20Stud.,\%206(4)\%208997,\%202016.pdf>. Acesso em: 27 julho 2017.

KOLB, D. Experiential learning: experience as the source of learning and development, Prentice-Hall, Englewood Cliffs, N .J., 1984.

LIKER, J. The Toyota Way: 14 management principles from the world's greatest manufacturer. McGraw-Hill, New York, 2004.

LIKER, J.; MEIER, D. The Toyota Way Field book: a practical guide for implementing Toyota's 4Ps. McGraw-Hill, New York 2006.

LITZINGER, T. A.; LEE, S. H.; WISE, J. C.; FELDER, R. M. A psychometric study of the index of learning styles $($ ). Journal of Engineering Education, v. 96, n. 4, p. 309, 2007. https://doi.org/10.1002/j.2168-9830.2007.tb00941.x

LOBAUGH, M. Lean manufacturing: a unique approach to educating students, v. 10, n. 1, 2005.

MARTENS, I.; COLPAERT, J.; DE BOECK, L. Lean learning academy: an innovative learning concept in engineering curricula. IHEPI 2010 Conference Paper, 2010.

MEYERS, L.; GAMST, G.; GUARINO, A. Applied multivariate research. Sage Publications, Thousand Oaks, 2006.

MONTGOMERY, D. Design and analysis of experiments. 8th ed. Wiley, New York, 2013. 
MURMAN, E.; MCMANUS, H.; CANDIDO, J. Enhancing faculty competency in lean thinking bodies of knowledge. $3^{\text {rd }}$ International CDIO Conference, 2007.

http://hdl.handle.net/1721.1/83592

PEDLER, M.; ABBOTT, C. Lean and learning: action learning for service improvement. Leadership in Health Services, v. 21, n. 2, p. 87-98, 2008.

https://doi.org/10.1108/17511870810870538

PETER, G. Hands-on graduate courses in lean manufacturing (LM) emphasizing green and total productive maintenance (TPM). American Society of Mechanical Engineers, 2010 International Mechanical Engineering Congress and Exposition, p. 357-365, 2010. http://dx.doi.org/10.1115/IMECE2010-37921

PETERS, F.; POTTER, L.; MIN, K. Developing students' understanding of global issues through lean manufacturing. $\mathbf{3 8}^{\text {th }}$ Annual Frontiers in Education Conference, IEEE, 2008. http://dx.doi.org/10.1109/FIE.2008.4720592

PUJI, R.; AHMAD, A. Learning style of MBTI personality types in history learning at higher education. Scientific Journal of PPI-UKM, v. 3, n. 6, p. 289-295, 2016. Disponível em < http://www.kemalapublisher.com/index.php/ppi-ukm/article/view/224>. Acesso em: 27 julho 2017.

RAMSDEN, P. Learning to teach at higher education. Taylor and Francis, London, 2003.

RULTEGDE, S. What do teachers know about differentiated instruction? Tese

(Doutorado) - Graduate Faculty of the Louisiana State University and Agricultural and Mechanical College, Southern University, 2016.

SANTOS, D.; SILVA, C. Evaluation of students' general perceptions of problem-based learning in a computer engineering program in Brazil. Problems of Education in the $21^{\text {st }}$ Century, v. 68, p. 74-83, 2015.

SEDDON, J.; CAULKIN, S. Systems thinking, lean production and action learning. Action Learning: Research and Practice, v. 4, n.1, p. 9-24, 2007.

http://dx.doi.org/10.1080/14767330701231438

SHAH, R.; WARD, P. Defining and developing measures of lean production. Journal of Operations Management, v. 25, n. 4, p. 785-805, 2007.

https://doi.org/10.1016/j.jom.2007.01.019

SHEPPARD, S.; MACATANGAY, K.; COLBY, A.; SULLIVAN, W.; SHULMAN, L.

Educating engineers: designing for the future of the field. Jossey-Bass, Hoboken, NJ, 2008.

SPEAR, S.; BOWEN, H. Decoding the DNA of the Toyota production system. Harvard Business Review, v. 77, p. 96-108, 1999.

TABACHNICK, B.; FIDELL, L. Using Multivariate Statistics. 5th ed., Pearson, Upper Saddle River, NJ, 2013.

TORRES, P.; STEPHENS, M. Improving production performance through lean manufacturing techniques \& education in lean concepts. 2005 American Society for Engineering Education Annual Conference \& Exposition, 2005. 
TORTORELLA, G.; FOGLIATTO, F. Method for assessing human resources management practices and organizational learning factors in a company under lean manufacturing implementation. International Journal of Production Research, v. 52, n. 15, p. 4623 4645, 2014. http://dx.doi.org/10.1080/00207543.2014.881577

TORTORELLA, G.; MARODIN, G.; MIORANDO, R.; SEIDEL, A. The impact of contextual variables on learning organization in firms that are implementing lean: a study in Southern Brazil. The International Journal of Advanced Manufacturing Technology, v. 78, n. 912, p. 1879-1892, 2015. https://doi.org/10.1007/s00170-015-6791-1

TORTORELLA, G.; VIANA, S.; FETTERMANN, D. Learning cycles and focus groups: A complementary approach to the A3 thinking methodology. The Learning Organization, v. 22, n. 4, p. 229-240, 2015. https://doi.org/10.1108/TLO-02-2015-0008

TOVAR, E.; WARSHAWSKY, N. Use of a problem-based learning exercise to teach the lean 8-step problem-solving method. Nurse Educator, v. 40, n. 2, p. 101-104, 2015. https://doi.org/10.1097/NNE.0000000000000124

UNIVERSITY Of KENTUCKY. True Lean. Lean Systems Program, Disponível em: $<$ https://www.lean.uky.edu/>, Acesso em: 20 dez. 2016.

VAN TIL, R.; SENGUPTA, S.; FLIEDNER, G.; TRACEY, M.; YAMADA, K. Teaching Lean manufacturing principles using an interdisciplinary project featuring industrial/academic cooperation. Frontiers in Education Conference, v.35, n. 3, 2005.

https://doi.org/10.1109/FIE.2005.1612258

WAN, H.; LIAO, Y.; KURIGER, G. Redesigning a lean simulation game for more flexibility and higher efficiency. 2012 American Society for Engineering Education Annual Conference \& Exposition, 2012.

WHITE, H. Problem-based learning. Speaking of Teaching, v.11, n.1, p. 1-7, 2001.

WOMACK, J.; JONES, D. Lean solutions: how companies and customers can create value and wealth together. Simon and Schuster, New York, 2009.

ZHANG, J.; OGAN, A.; LIU, T.; SUNG, Y.; CHANG, K. The influence of using augmented reality on textbook support for learners of different learning styles. Mixed and Augmented Reality (ISMAR), 2016 IEEE International Symposium on (pp. 107-114), IEEE, 2016.

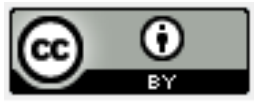

Artigo recebido em 06/01/2017 e aceito para publicação em 25/07/2017 DOI: http://dx.doi.org/10.14488/1676-1901.v18i1.2682 